STUDI

FRANCESI

\section{Studi Francesi}

Rivista quadrimestrale fondata da Franco Simone

143 (XLVIII | II) | 2004

Varia - fasc. II - maggio-agosto 2004

\title{
Luca Pierdominici, La bouche et le corps. Images littéraires du Quinzième siecle français
}

\section{Maria Colombo Timelli}

\section{(2) OpenEdition}

1 Journals

\section{Édition électronique}

URL : https://journals.openedition.org/studifrancesi/39032

DOI : 10.4000/studifrancesi.39032

ISSN : 2427-5856

Éditeur

Rosenberg \& Sellier

\section{Édition imprimée}

Date de publication : 1 décembre 2004

Pagination : $342-343$

ISSN : 0039-2944

\section{Référence électronique}

Maria Colombo Timelli, «Luca Pierdominici, La bouche et le corps. Images littéraires du Quinzième siecle français ", Studi Francesi [En ligne], 143 (XLVIII | II) | 2004, mis en ligne le 30 novembre 2015, consulté le 19 mai 2021. URL : http://journals.openedition.org/studifrancesi/39032 ; DOI : https://doi.org/

10.4000/studifrancesi.39032

Ce document a été généré automatiquement le 19 mai 2021.

\section{(c) $(1) \odot$}

Studi Francesi è distribuita con Licenza Creative Commons Attribuzione - Non commerciale - Non opere derivate 4.0 Internazionale. 


\title{
Luca Pierdominici, La bouche et le corps. Images littéraires du Quinzième siecle français
}

\author{
Maria Colombo Timelli
}

\section{RÉFÉRENCE}

LUCA PIERDOMINICI, La bouche et le corps. Images littéraires du Quinzième siecle français (Préface de Jean Dufournet), Paris, Honoré Champion, 2003 («Bibliothèque du XV siècle», 65), $285 \mathrm{pp}$.

1 Cet ouvrage, qui constitue une sorte de complément français au recueil d'études en langue italienne que l'A. a publié en 2002 (Prose francesi del XVsecolo. Università degli Studi di Macerata: cf. «Studi Francesi», XLVII, 2, 2003, pp. 420-21) réunit une dizaine d'articles consacrés à la littérature française $\mathrm{du} \mathrm{XV}^{\mathrm{e}}$ siècle, et notamment aux œuvres d'Antoine de La Sale, aux Cent Nouvelles nouvelles, au Testament de Villon, aux Arrêts d'Amour de Martial d'Auvergne et aux Quinze joies de mariage. Ces travaux, qui remontent aux années 1992-2001, sont organisés en trois sections. La première partie, sur Antoine de La Sale, comprend deux études complémentaires, l'une centrée sur les deux traités didactiques, $L a$ Salade et La Salle, l'autre sur le Saintré ('A la verité me samble': l'esprit pédagogique de La Sale dans la «Salade» et la «Salle», pp. 19-38; «Chose vraye faict a doubter»: «Saintré» ou l 'invitation au mensonge, pp. 39-57). Si dans les œuvres expressément pédagogiques l'instruction aux jeunes princes, fondée sur l'érudition et sur la citation des auctores, peut glisser et glisse de fait vers les formes de la narration, et même de la narration personnelle, dans le Saintré le pédagogue se fait surtout conteur, dans un effort difficile, peut-être impossible aux yeux mêmes de l'auteur, de concilier idéal et réalité. La réaffirmation consciente des vieilles valeurs, présente aussi bien dans les traités que dans le roman, va de pair avec le regard désenchanté que La Sale sait porter sur son monde, un regard qui reconnaît les contradictions profondes de la vie de la cour, et peut-être aussi de la vie de tout homme. La section consacrée aux Cent Nouvelles 
nouvelles compte trois essais, dont le premier est centré sur le corps (Le corps dans les $\mathrm{CNn}$, pp. 61-122). Sont analysées d'abord les mentions du corps, aussi bien pour les parties «nobles» que pour les parties «basses»: toutes sont nommées par des termes employés au sens propre, par des expressions figées, par des mots utilisés au sens figuré, ou encore par des circonlocutions ou des euphémismes. Dans ce domaine, l'auteur donne la preuve de sa fantaisie verbale, et surtout il révèle sa propre vision, souvent ironique, des personnages et du monde qu'il met en scène. Cependant l'obscénité n'est pas le souci principal de l'acteur, le détail scabreux n'apparaissant que lorsqu'il est fonctionnel au récit. L.P. traite ensuite des fonctions du corps, en passant en revue les cinq sens de l'homme, qui tous entrent dans le jeu de la recherche du plaisir. Sur le plan culturel, dans les $\mathrm{CNn}$ le corps s'avère le moyen de la transgression des lois morales et de la mise en cause du code courtois. Le thème du corps, véritable protagoniste des récits selon l'A., dévoile la tendance à la rupture et à l'ironie, mais surtout il révèle une tentative réussie de gommer la peur de la mort. Les demières réflexions portent sur le "corps protagoniste»: décrit de façon détaillée, en position statique ou en mouvement, le corps structure le temps et l'espace du conte, où tout dépend de sa présence; il est, selon L.P., en même temps objet d'analyse et sujet de l'œuvre (p. 119). En conclusion, le corps constitue un thème massivement exploité dans le recueil; sur le plan narratif, il constitue le véritable moteur de l'action; surtout, il réflète l'importance que lui accordent les hommes du Moyen Age finissant.

2 Le style direct, étudié dans les douze premières nouvelles, fait l'objet d'un deuxième chapitre (Le style direct dans les CNn, pp. 123-146). Sont d'abord analysées les interventions de l'auteur, signalant sa présence par des appels au lecteur (qui peuvent comporter de véritables marques d'oralité), par des formules attestant la véracité de ses contes, par des commentaires où il s'exprime directement. L'examen des discours en style direct prend en compte les modes d'introductions (verbes introducteurs ou incises), les verbes utilisés, quelle que soit leur position (limités à dire, demander, respondre), l'ordre des mots dans les incises, les termes d'adresse (type et fréquence des appellatifs, leur position), les possibles «emboîtements discursifs» (un personnage s'exprimant en style direct rapporte à son tour les paroles de quelqu'un). Une telle analyse, qui se situe au croisement de l'étude strictement linguistique et de celle du style, prouve la variété et par là la richesse du recueil. Suit l'examen des deux formes que peut assumer l'expression indirecte de la parole: le style indirect et le style indirect libre (Les styles indirects dans les CNn, pp. 147-159). L.P. analyse premièrement les verbes introducteurs (plus variés que pour le style direct, ils comprennent des verbes d'opinion, des formules variées, mais on relève aussi des phrases à verbe sousentendu), puis les conjonctions introductives (que, comment, de pour les propositions infinitives); en particulier, il constate la redondance de la conjonction que, phénomène fréquent dans la prose $\mathrm{du} \mathrm{XV}^{\mathrm{e}}$ siecle. Quant au style indirect libre, il garde quelques traces d'oralité; il est parfois difficile à délimiter sur le pian linguistique, et on peut y déceler même des interventions du narrateur. En conclusion, selon les mots de L.P., on pourrait considérer le recueil dans son ensemble "comme un discours non introduit» (p. 159), un long discours en style indirect libre, dominé par la voix de l'auteur.

3 L'étude consacrée au Testament (pp. 163-210) s'appuie sur le constat préliminaire que le corps, celui du poète et celui des autres personnages évoqués, occupe une place importante dans l'œuvre maîtresse de François Villon. Dans une première section, L.P. prend en compte les mentions du corps (cf. la liste exhaustive donnée aux pp. 209-210); 
en effet, toutes les parties du corps sont citées dans le Testament énoncées directement ou rappelées par des allusions ou des euphémismes, ou encore insérées dans des expressions figurées ou des jeux de mots; encore, dans l'opposition corps-cuer, Villon semble accorder une plus grande importance au premier, entre autres par le nombre des occurrences relevées; enfin, alors que les allusions à son propre corps sont moins nombreuses et correspondent plutôt à l'image idéale que le poète veut transmettre de lui-même, les allusions au corps de ses légataires sont beaucoup plus fréquentes et réalistes. La deuxième partie conceme les fonctions que Villon attribue au corps, fonctions physiques (recherche du plaisir, sexuel et alimentaire, crainte de la douleur sous ses aspects matériels - pauvreté, vieillesse, mort - et moraux), mais aussi fonction poétique: sous cet angle, non seulement les dons se rapportent au corps des destinataires, mais certains détails et allusions contribuent à la construction de situations existentielles précises. En conclusion, selon L.P., le corps du poète est directement engagé dans le Testament, son traitement est révélateur en même temps de la sensibilité du $\mathrm{XV}^{\mathrm{e}}$ siècle et de la vision personnelle du poète, pour lequel le corps constitue le point de contact irremplaçable entre son monde fictif et la réalité.

L'article Conter et juger dans les «Arrêts d'Amour» de Martial d'Auvergne (pp. 211-225) a déjà été publié dans les "Quaderni di Filologia e Lingue Romanze - Ricerche svolte nell'Università di Macerata», terza serie, 15, 2000, pp. 175-192; et dans «FifteenthCentury Studies», 28, 2003, pp. 199-211. Nous en avons rendu compte dans «Studi Francesi», XLVI, 138, 2002, p. 675. Une dernière contribution est consacrée aux Quinze joies de mariage (Lire la joie, ou l'efflorescence du texte dans les «Quinze joies de mariage» pp. 227-243). Malgré une structure rigide qui lui vient du modèle dont il se veut la parodie, ce recueil réunit des textes dynamiques et ondoyants: l'auteur semble hésiter entre les histoires à raconter, parfois il propose des déroulements divers, il cherche des formes d'expression et un style adéquats dans des altemances que le moyen français admet encore (par exemple dans l'alternance des temps verbaux). Mais surtout l'auteur hésite quant au mode d'énonciation de ses textes, démonstratifs et narratifs à la fois; il pratique ainsi une «écriture intermittente» (p. 243), qui est à la source du texte même, un texte vivant, efflorescent et, au fond, «jubilatoire».

5 La Bibliographie réunie par L.P. est très vaste (pp. 245-270); elle organise en sections séparées les đuvres (mss., éditions anciennes, éditions modernes et/ou traductions) et les Etudes (la subdivision interne, fondée en partie sur les sujets, parfois sur la forme monographies, chapitres spécifiques, articles- n'est pas toujours très claire). Le volume se clôt avec deux compléments: l' Index des noms et des titres (pp. 271-274) et l'Index des notions (pp. 275-281). 